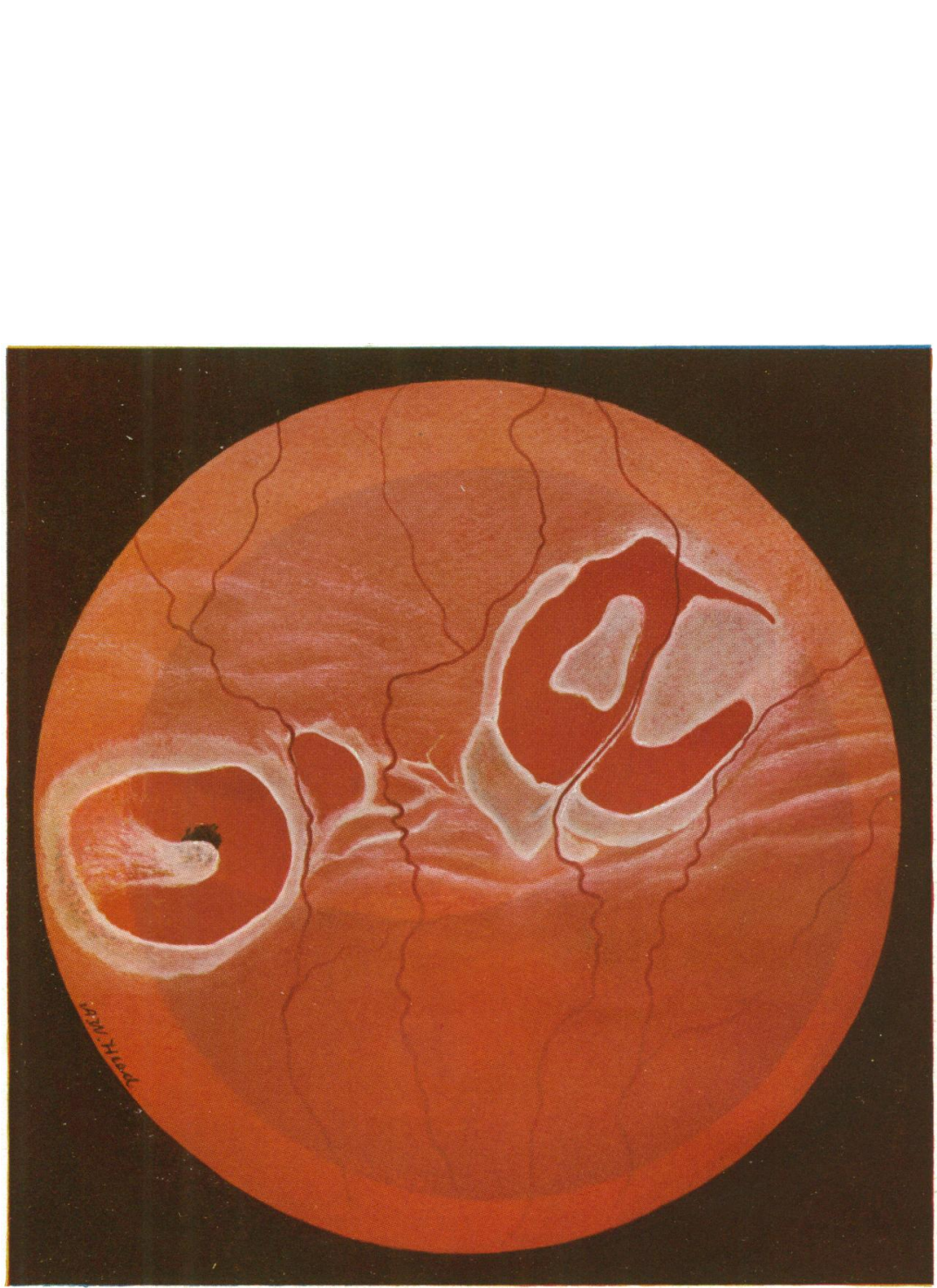

Plate I.

Holes in a detached retina. 


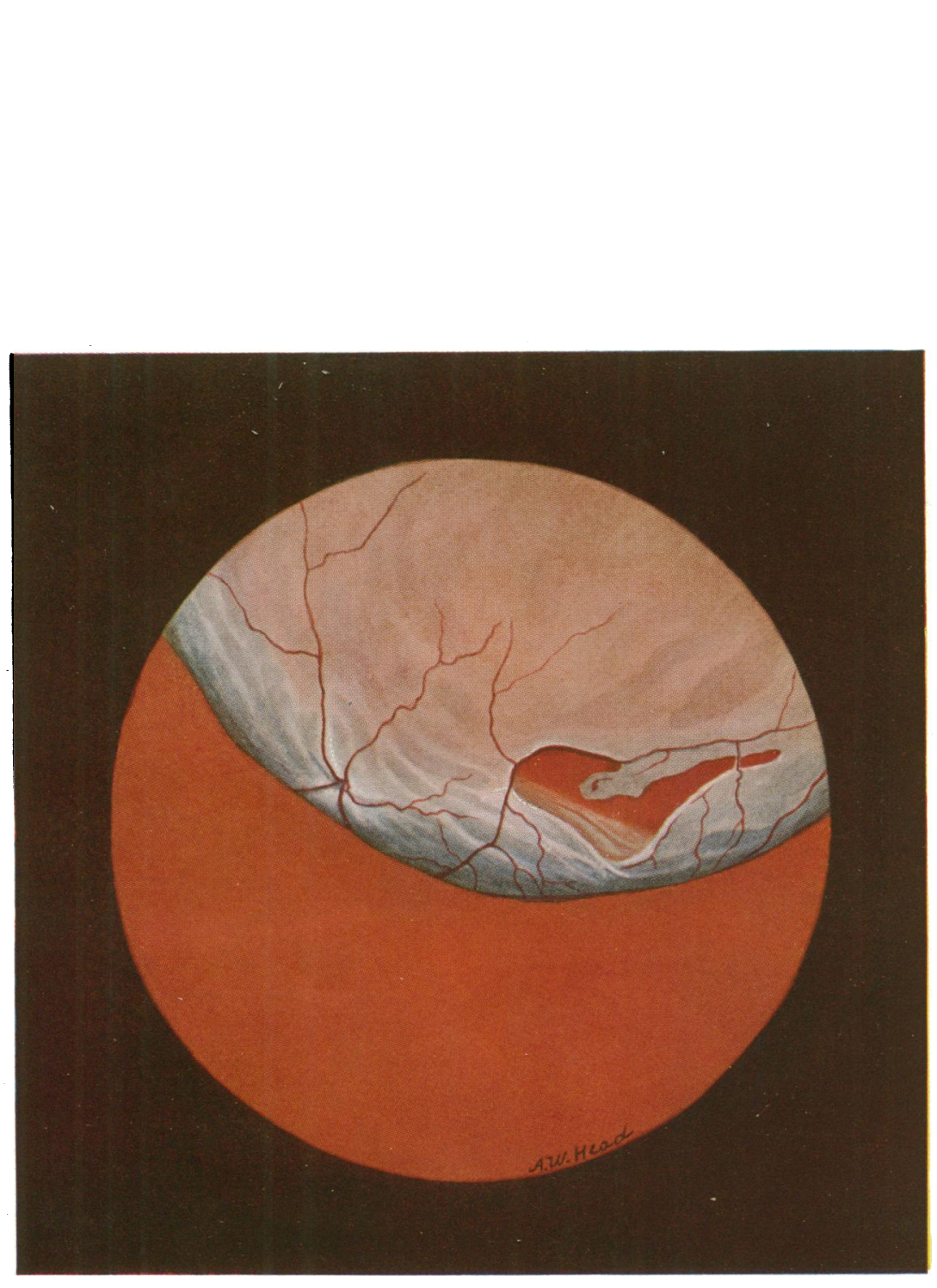

Plate II.

Globular detachment above in which there is a large retinal rent, through which not only the choroid but also outer surface of retina is visible. 
HOLES IN THE RETINA AND THEIR CLINICAL SIGNIFICANCE*

BY

Sir William Lister

LONDON

I HAVE chosen as the subject of my paper, one on which I fear I cannot throw much new light, but it is one to which, in my opinion, too little attention is paid clinically. My subject is "Holes in the Retina and their Clinical Significance," and my remarks will be divided under three headings :-

I. The various clinical varieties of holes in the retina.

II. Their aetiology.

III. Their clinical significance.

\section{Clinical varieties}

For convenience we may divide holes in the retina into two main groups-macular holes and peripheral holes, and subdivide each of these under the two headings of traumatic and non-traumatic.

(1) Macular holes. We are all familiar with the so-called " macular holes"; they occur both after trauma and without trauma.

(a) Traumatic. The greater number of macular holes follow a blow on the anterior part of the eye from non-perforating missiles,

"Delivered before the Ophthalmic Section of the Academy of Medicine, Toronto, October, 1923. 
such as stones, fists, etc. These cases have been well described and illustrated by various observers.

As seen with the ophthalmoscope, the hole may be single, round or oval, or there may be two or more holes. The hole has a sharply punched out appearance, its margin being whiter than the surrounding retina and occasionally decorated with small rounded masses. The edge is sometimes raised, indicating a slight detachment, but any great degree of separation is very rare. The centre of the hole is bright red and frequently exhibits some pigmentary disturbance or glistening dots.

(b) Non-traumatic. The appearance of a hole at the macula is also found in cases where no history of a blow is obtainable, and in which retino-choroidal degeneration is the most probable cause. Whether these are true holes or whether the appearance of a hole is due to cystic degeneration of the retina will be discussed later.

(2) Peripheral holes. (a) Traumatic. We have all seen cases of holes situated in the periphery of a detached retina, following a blow. These are large oval or circular rents or they may be slit-like or shaped like a horse-shoe and in many of the latter the central portion is bent inwards. Occasionally large gaps in the retina are found situated so far forward that only the posterior margin of the rent is visible. These are probably due to tearing of the retina from the ora serrata. Traumatic peripheral holes are usually associated with considerable detachment.

In my own experience peripheral holes due to trauma without detachment are very uncommon. I saw one case some years ago, that of a boy who was struck by a piece of paving material on the outer side of the sclerotic, sufficiently hard to make the eye bleed. His sight was impaired for a few days but later full acuity was restored. It was only after dilating the pupil and examining the extreme periphery that a hole in the retina was discovered on the outer side. It was so far forward that only the posterior margin of the rent was visible, the anterior margin being shut off from observation by the edge of the pupil and thus very possibly the tear in this case was at the ora serrata.

The portion of the edge of the rent which could be seen was white and slightly raised and through the gap the choroid was seen a bright red ; there was no visible detachment of the retina and the field was full. This case had a special interest from the medicolegal aspect, for the boy was on duty when he was struck and was therefore entitled to compensation for injury. His sight, however, was perfect and therefore at the time compensation only for loss of work could be claimed. On the other hand, with this hole, detachment might occur at any moment which would have caused 
complete loss of sight in this eye, when he would have been entitled to a heavy compensation. I have lately tried to trace this boy to learn the present condition, but failed as he had gone to New Zealand.

(b) Non-traumatic. Holes associated with choroiditis or contraction of the vitreous are according to my experience more frequently discovered during pathological investigation than with the ophthalmoscope.

\section{Aetiology of Holes}

There are some who have suggested that in every detachment there is a hole in the retina. Such a view can hardly be accepted when the different consistency of the fluid in the inter-retinal space is noted in different cases.

Though in some cases of detachment, where no hole is discoverable, the consistence of the inter-retinal fluid is clear and thin after fixation and similar in appearance to that of the degenerated vitreous, yet I think we shall all agree that in the majority of cases of detachment without a hole, the inter-retinal fluid after fixation sets into a firm, jelly-like mass. On the other hand, in every case in which a hole has been found, the consistency of the inter-retinal fluid after fixation is exactly similar to that of the fluid vitreous. If the fluid in the inter-retinal space has free communication with the vitreous by means of a hole it would seem incredible that the two should not mix. One is, therefore, forced to hold the view that when, after fixation, a solid coagulum is found in the inter-retinal space, there cannot be a hole.

The cases where the inter-retinal fluid is, of similar consistency to the vitreous and no hole is found, are probably either (1) those in which the observation is incomplete, the hole being obscured by a fold in the retina or so small that it is undetected, or (2) those in which a change has taken place in the proteid molecules of the inter-retinal fluid, breaking this up into more readily diffusible substances.

From the variety of the position of holes and of their associated conditions, it would be inferred that there must be several different causes which bring them about.

Traumatic Macular Holes. A considerable amount of literature has appeared on the cases of so-called macular holes following trauma giving reports of the pathological investigations. That actual holes occur at the macula is proved by their having been found microscopically. Whether these holes were caused directly by the blow or whether the retina had first undergone a cystic degeneration as the result of a blow and the hole was caused subsequently by the rupture of the cyst walls is as yet uncertain. 
Cystic degeneration is frequently seen in uninjured eyes in the forward portion of the retina near the ora serrata, and, though less common in the central region, large cysts at the macula with very thin walls are occasionally met with. It has yet to be determined whether all cases in which the ophthalmoscope reveals the appearance of a hole have definite gaps in the retina, or whether in some the ophthalmoscopic appearance of a hole may be brought about by a cyst of the retina alone without rupture.

Unfortunately the justification for removing eyes with the ophthalmoscopic appearance of a macular hole is so rare that little opportunity is offered for enlarging our knowledge.

A reasonable explanation of this macular condition would seem to be, that since most macular holes are due to a blow on the anterior part of the eye, the macula will lie at the point of contrecoup. " Not only is the macula the most delicate part of the retina but it is at this spot that the rending force of the pressure waves starting from the site of impact will be most felt, as I have described in a paper on ruptures of the sclerotic at the point of contrecoup. This rending force in some eyes may cause an actual rupture of the retina, in others it may be followed by cystic degeneration, and, in a proportion of these cases rupture of the cyst walls takes place.

Macular holes are, however, also seen as a result of lateral blows on the eye. These were seen during the war in a certain number of cases, where missiles passed through the side of the orbit without actually striking the eye. With these injuries it was the rule to find the concussior changes situated adjacent to the site of impact, but in some cases changes were also found at the macula. In the majority of these the macular lesion appeared merely haemorrhagic, but in a certain proportion there were seen the socalled "holes." Such macular disturbance, not at the point of contrecoup but more in the region of the equator round the line of impact, is difficult to explain, but may possibly be accounted for by the fact that the macula, being the most delicate part of the retina, is more liable to be injured by sudden increase of intraocular pressure than other parts of the retina.

Peripheral Traumatic Holes. Peripheral holes may certainly occur adjacent to the site of impact as in the case I have already cited, where the foreign body struck the outer side of the eye, and the hole in the retina was found in the outer periphery. This corresponds with our experience during the war of concussion injury of the eye, where on microscopic examination the retina was frequently found ruptured adjacent to the site of impact.

But peripheral holes also occur after trauma in the protected parts of the eye, where they could hardly be due to direct impact. It is possible that these ruptures of the retina correspond with the 
ruptures of the sclerotic seen in civil practice following direct impact from slowly moving objects, and are due to the blow causing distension in the equator round the line of impact. The sclerotic containing elastic fibres is more resilient and does not rupture so readily, but the retina having no elastic fibres gives way before pressure. If this be the cause, however, it is surprising that these cases of peripheral rents, not occurring at the site of impact, are not more frequently associated with macular holes also.

Non-traumatic holes. Passing now to the non-traumatic holes, we find various theories put forward to account for their occurrence.

(1) We must certainly place cystic degeneration as a likely cause.

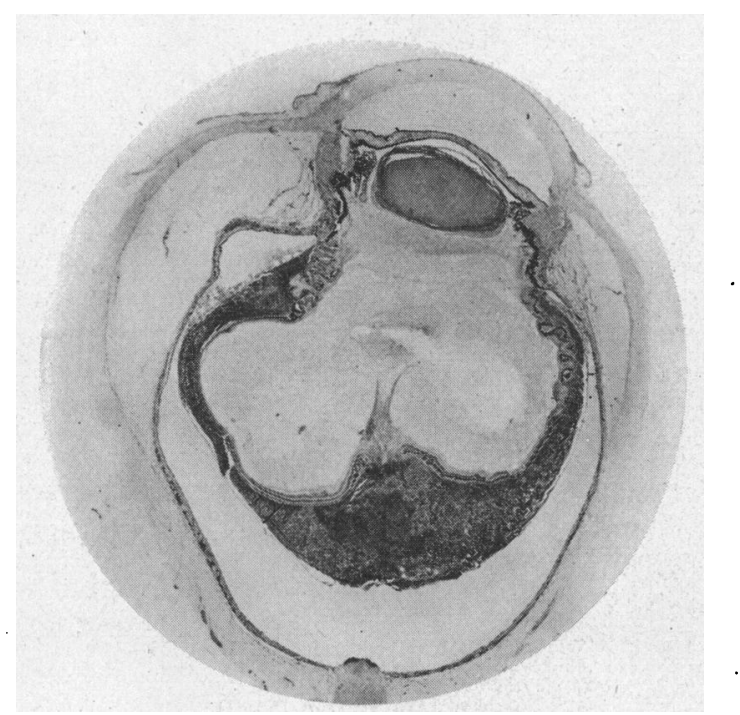

FIG. 1.

A large cyst or numerous small cysts having formed from whatever cause, gradual expansion of the cyst with consequent thinning of the walls may bring about rupture, or the latter may be determined by some slight trauma from without or traction of adherent vitreous from within.

I have recently seen a case of superior detachment in a myope in which there were several holes, over two of which retinal vessels coursed. In one hole an island of retina appeared to lie unattached, though there may have been some point of connection with the rest of the retina which was invisible.

I cannot offer a wholly satisfactory explanation of this, to me very unusual, case, but would suggest that it is probably due to rupture of cysts. 
(2) Raehlman's osmotic diffusion theory. When an intact retina separates a highly albuminous exudate in the inter-retinal space from a watery vitreous, diffusion from the vitreous to the albuminous exudate might be expected to take place, which would increase the area of detachment. Raehlman suggests that by this diffusion of fluid the tension in the inter-retinal space may become so much greater than in the vitreous, that rupture of the retina takes place.

This theory of the formation of holes has several objections.

(i) Have we any evidence that osmosis takes place through the living and intact retina? Though theoretically it might take place, it appears very doubtful if much diffusion from within outwards actually occurs. For in long standing cases of detachment due to sarcoma of the choroid, where after fixation the vitreous remains clear and the inter-retinal fluid sets solid, it is obvious that the inter-retinal fluid remains more albuminous than the vitreous; one would have expected that if osmosis occurs to any marked degree, the two fluids would not remain so dissimilar.

(ii) Considering the extremely easy separation of the retina from its pigment epithelial layer, one would expect that should a difference of pressure on its two sides occur, it would merely lead to increase in the detachment. A difference of pressure on the two sides of the retina sufficient to cause rupture, could only arise if the retina were pegged down by previous retino-choroiditis, or if the detachment was nearly complete.

Therefore it seems probable that holes in the slighter degrees of detachment and in the absence of choroiditis can hardly be explained by Raehlman's theory.

(3) Elschnig's Theory. Elschnig explains the presence of holes. in the retina by the combination of detachment with a pegging out of the retina to the choroid by pre-existing areas of retino-choroiditis. If the detaching force, whether traction from within or pressure from without, be sufficient to tear the retina, holes will be produced where it is pegged out. On the other hand when the retina is too tough to tear, it will remain adherent to the choroid in a funnelshaped cord at these spots.

It is disappointing that in all the reported cases of holes due to pegging out of the retina by choroiditis, which I have come across, the accounts have been incomplete. Thus in Elschnig's first reported case there was a patch of retino-choroiditis seen ophthalmoscopically; and at a later examination a hole in the retina was found corresponding in position with the spot of retino-choroiditis, but the condition was not substantiated anatomically. In his second case there was a large hole in the retina and through it degenerated choroid was seen, but it was not proved that a piece of retina was torn out.

In Collins' two cases, patches of degenerated retina were found 
attached to the choroid but the holes in the retina were not seen, though they must have existed in the contracted retina.

In spite of the reports being thus incomplete it is quite evident that Elschnig's theory is a true explanation of certain cases.

A somewhat rare but allied condition illustrates his theory, namely total detachment of the retina. (Fig. 1.) In such cases though the hole is not seen except on microscopic section, the retina being contracted up into a mass behind the lens, the disc is found with no retina attached, showing that the latter must have been completely torn away.

A different result will occur according to the nature of the detaching force, i.e., whether there is "a pushing from without" by fluid secreted into the inter-retinal space, or by possible osmosis; or "a dragging from within" by an adherent and contracting vitreous.

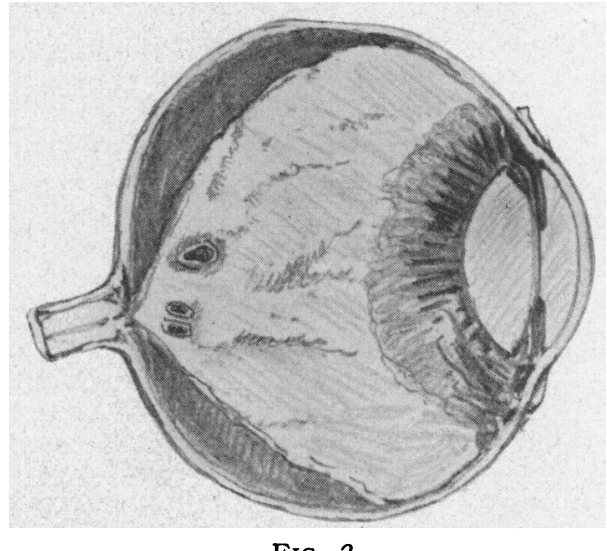

FIG. 2.

If the force is pushing from without, directly one hole occurs, equalisation of pressure on the two sides of the retina will be brought about and no further detachment or tearing will occur; that is to say only one hole can be produced by pushing from without. If, however, the detaching force is traction from within, then as many holes may be formed as there are points of attachment, provided the detachment is sufficiently extensive.

I examined the eye of a doctor who, when lowering his head to look down a microscope, ran a knife into his eye. There was a wound of the sclerotic at the limbus, the cornea was hazy with striate keratitis, the anterior chamber was deep, the iris muddy, the lens uninjured. The vitreous was shrunken to $1 / 6$ th of its normal size and there was haemorrhagic infiltration at the site of the wound, but it was in no way visibly attached to the retina. The retina was detached and in it were three circular punched out holes, one by $i$ tself and two together with the edges inverted. (Fig. 2.) Opposite 
the holes were small whitish spots on the choroid, while in several other places the retina was attached to the choroid. Sections were cut at the site of the holes and also where the retina was adherent.

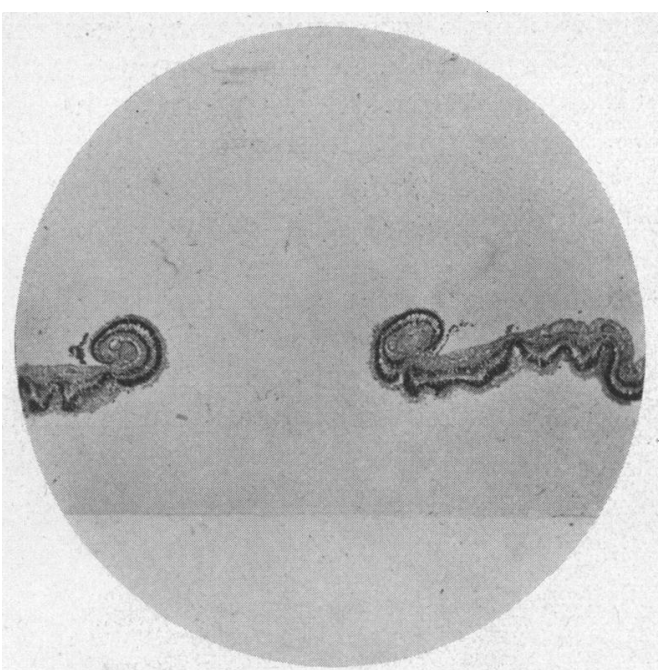

FIG. 3.

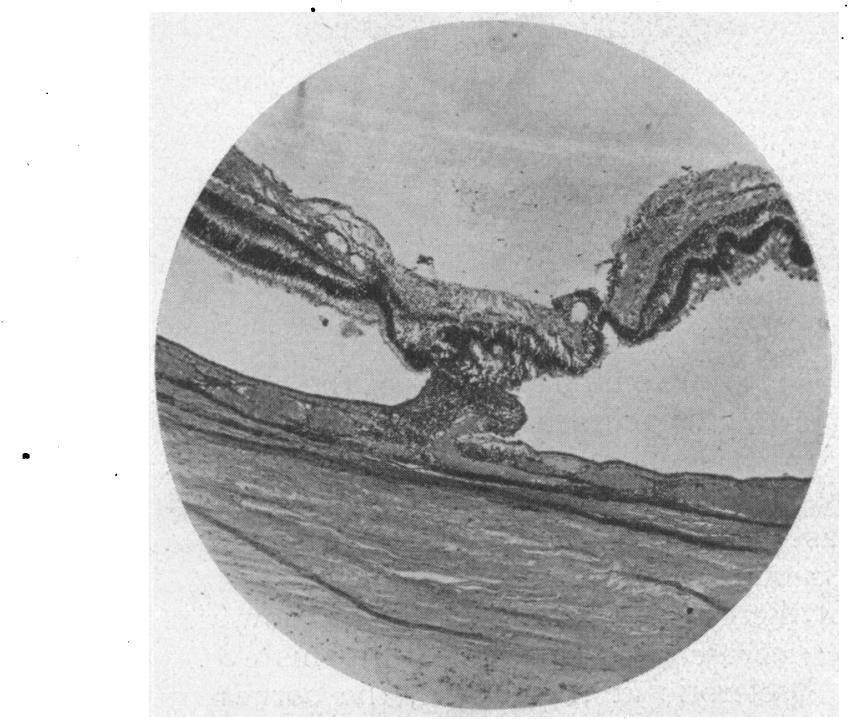

FIG. 4.

(Figs. 3, 4, 5.) In spite of serial sections being made I could find no trace of retina remaining adherent to the choroid opposite the holes; but there was some laminated new-formed fibrous tissue, which evidently gave rise to the white spots seen macroscopically. 
These fibrous patches were rich in cells similar to those which were attaching the retina to the choroid at the points of adhesion. (Fig. 6.) Though in this case the holes were obviously associated with choroiditis I am unable to decide whether the retina was "pushed in" or "pulled in." The vitreous was demonstrably contracted, but between the shrinking vitreous and the retina

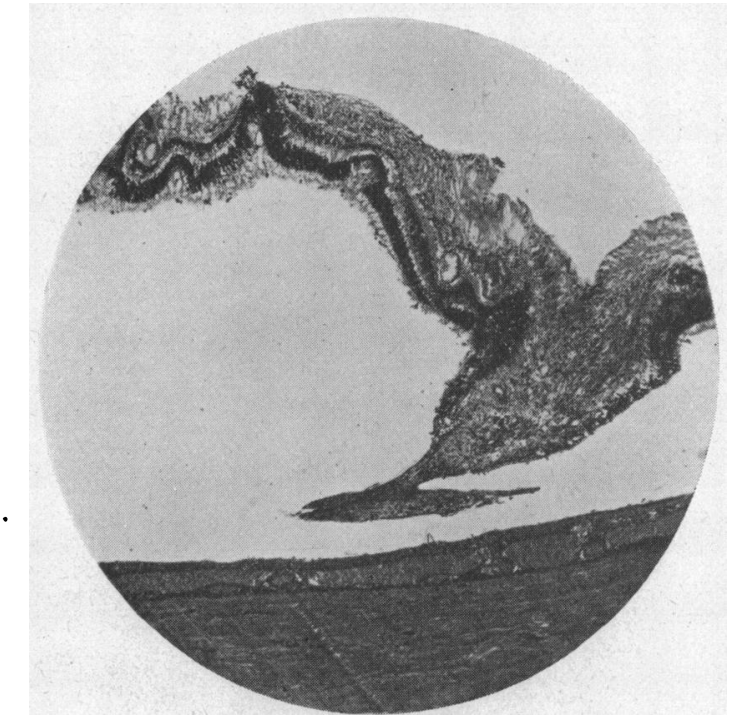

Fig. 5.

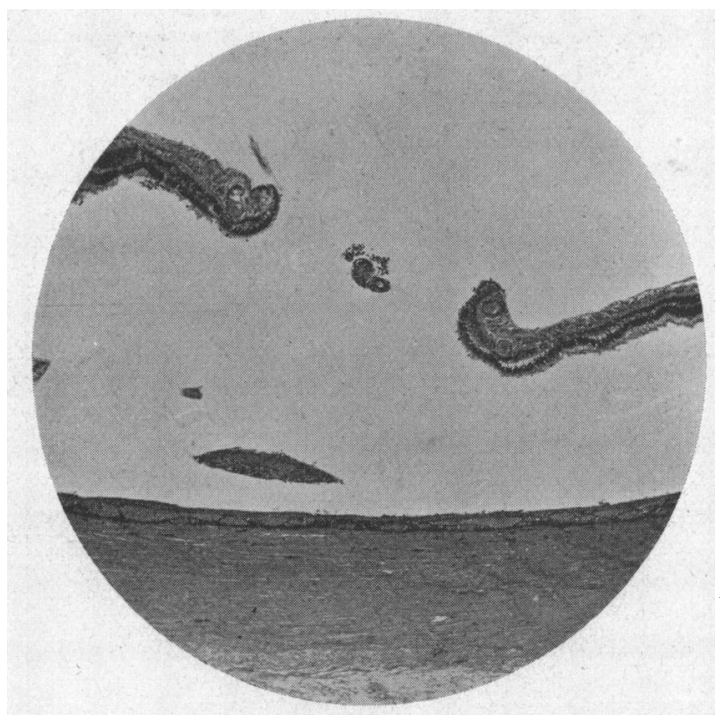

FIG. 6. 
there was a layer of clear limpid fluid; if therefore the retina was "pulled in," why were no points of adhesion of the vitreous to the retina discovered? While if it was "pushed in" how could the presence of three holes be accounted for?

The retina round one of the holes was much degenerated and cystic (Fig. 7) and therefore obviously very friable, so that very " slight traction would be sufficient to cause a rent. It is possible the vitreous had previously been adherent and dragged on the retina, causing it to tear, while at a later date the points of adhesion to the retina had given way.

Though unable to explain the actual mechanism, this case is

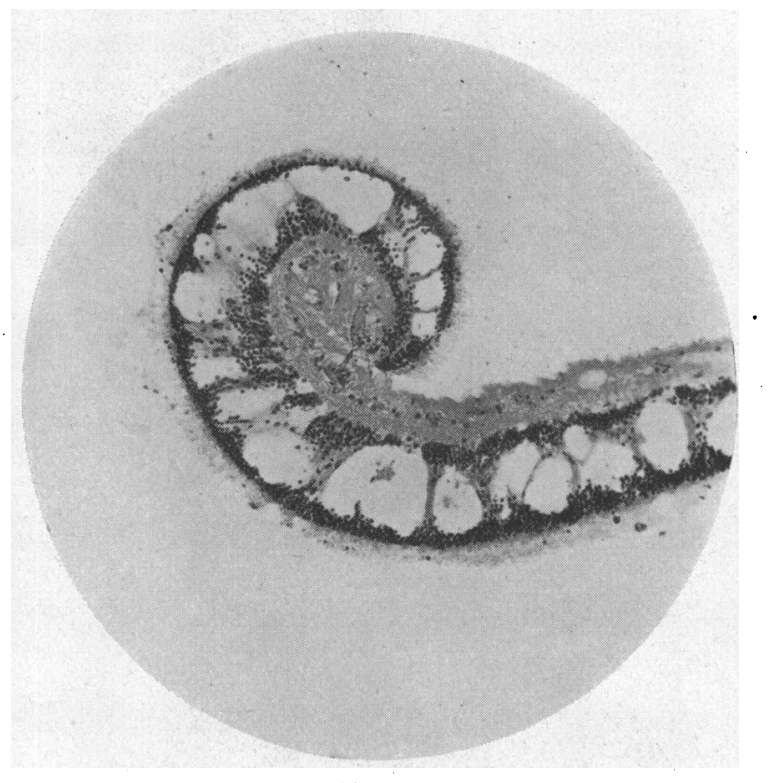

FIG. 7.

brought forward to illustrate the forms of holes associated with retino-choroiditis.

(4) Leber and Nordenson's theory of traction by fibrous bands in the vitreous. That macroscopic fibrous-bands in the vitreous attached to the retina may cause folding, crinkling and detachment of this membrane is well known; we have all seen cases of retinitis proliferans ophthalmoscopically, giving rise to this appearance, and the following specimens of traumatic retinitis proliferans demonstrate them. (Figs. 8, 9.)

Such fibrous bands most frequently follow a haemorrhage from the retina into the vitreous from trauma, or are possibly-as Flemming suggests-associated with some toxic inflammatory condition; the vitreous thus becomes adherent to the retina at 
the site of the haemorrhage, and, on its shrinking and becoming separated elsewhere from the retina, the latter is dragged on and becomes detached. (Fig. 10.)

In other cases attachment of the vitreous to the retina follows plastic cyclitis when fibrinous exudate is poured out into the vitreous which subsequently contracts. The following sections of a case

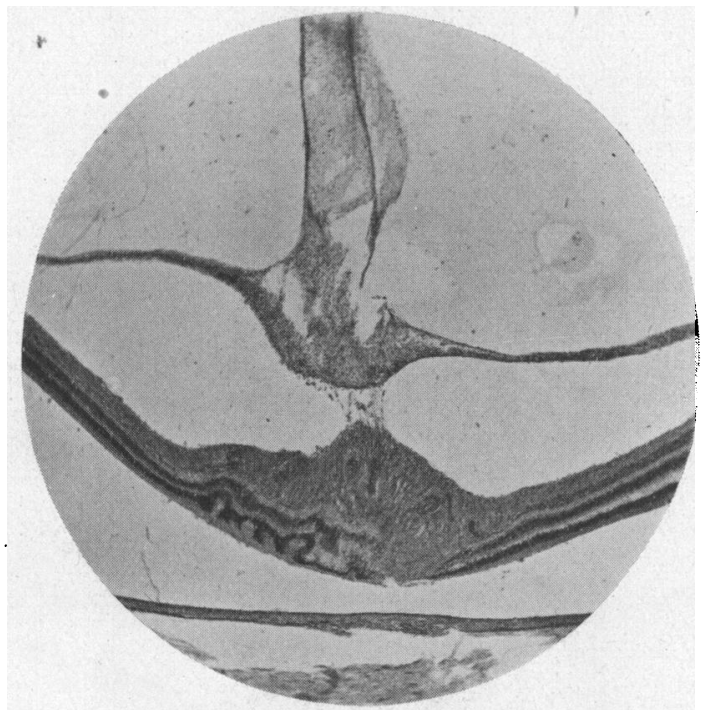

FIG. 8

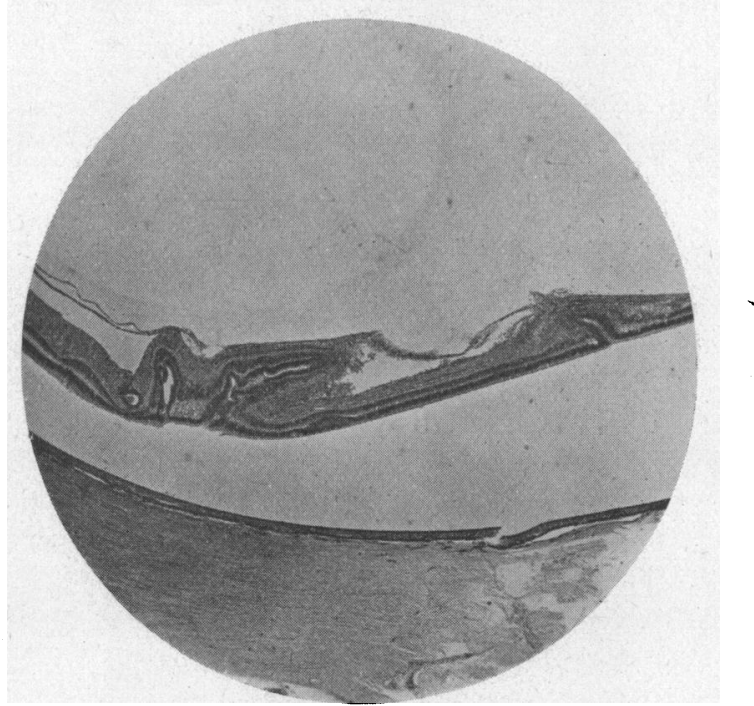

FIG. 9. 
of plastic irido-cyclitis showing fibrinous exudate in the vitreous, detachment of the retina, and detachment of the choroid demonstrate the festooning of the retina between its points of adherence to the . choroid caused by the contraction of the vitreous. (Figs. 11, 12.) If the adhesions, either of the vitreous to the retina, or of the retina

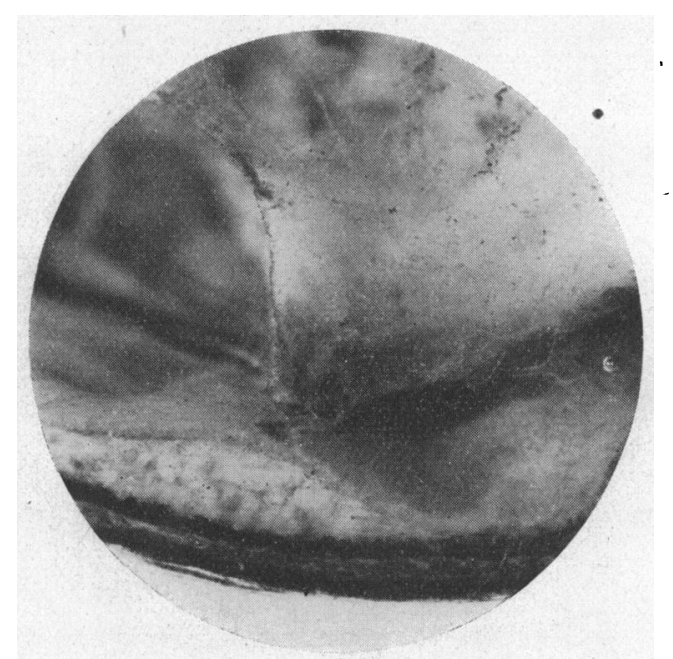

FIG. 10.

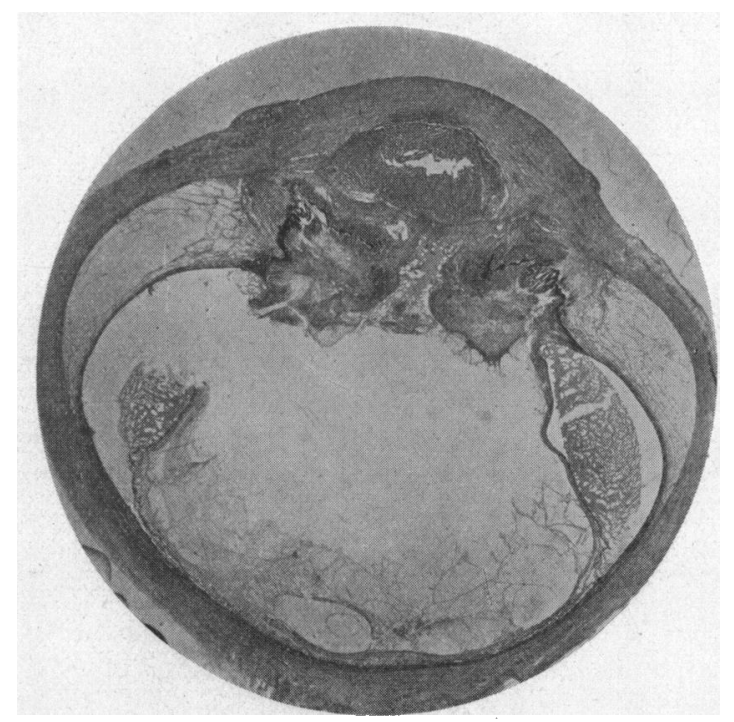

FIG. 11. 
to the choroid had been stronger, it is easy to see that holes could have been formed, either according to Leber's or Elschnig's theories.

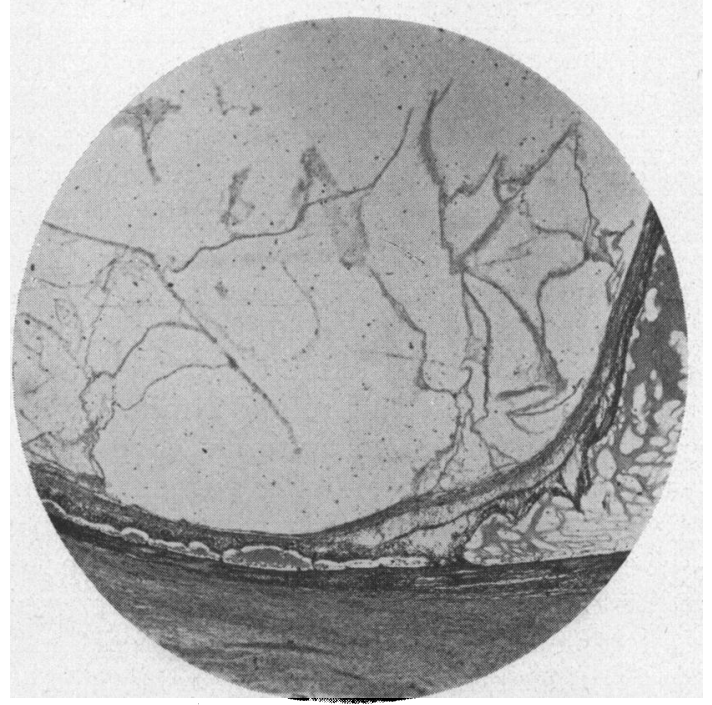

FIG. 12.

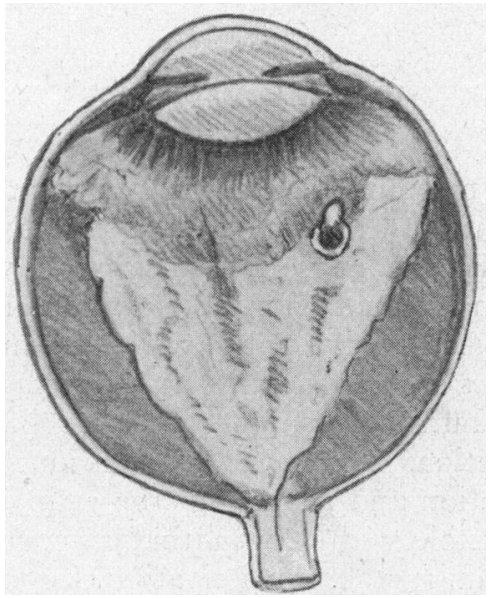

FIG. 13.

To show that a fibrous and shrinking vitreous adherent to the retina may cause holes apart from choroiditis, I may quote one of Lawford's cases, a woman aged 60 , whose sight failed suddenly. Ophthalmoscopically no details were visible. There was only a red 
fundus reflex downwards and down and out on focal illumination, and there was much floating vitreous opacity. A growth was suspected and the eye excised, and I had the good fortune to examine it. (Fig. 13.) The anterior part of the eye was normal, the vitreous was brownish and contracted to less than half its normal bulk and fibrous in consistency. Between the vitreous and the posterior part of the retina was a space filled with clear fluid, but from the ora serrata to near the region of the equator the vitreous was adherent to the retina. The retina was detached all round from the ora serrata to the disc, and the subretinal fluid was clear. At one spot near the ora, the retina was so much puckered and adherent to the vitreous that I suspected the presence of a foreign body. This was, however, excluded by X-ray examination. At one situation close to the junction of the retina and shrinking vitreous there was a hole in the retina $2 \mathrm{~mm}$. in diameter. Its edges were inverted and from its anterior edge there projected a flap, which was firmly adherent to the vitreous. Could this flap have been reflected back into place, it would have turned the hole into a horse-shoe shaped rent. Careful search failed to reveal any sign of choroiditis or disturbance of the retinal or choroidal pigment opposite the hole or elsewhere. This case appears to be obviously one of a hole in the retina torn by an adherent and shrinking vitreous.

Sometimes we see with the ophthalmoscope horse-shoe shaped rents in which the central flap is turned inwards and has some thickened vitreous attached to it. Such a condition strongly suggests that an adhesion of vitreous has taken place, which by dragging on the retina has brought about a rent.

It is thus clear that macroscopic fibrous bands can cause both detachment and rents in the retina ; but how are we to explain the numerous cases of holes met with clinically in which there is no history of trauma, no sign of choroiditis which might peg out the retina, and no marked opacity of the vitreous-merely a few webs or floating dust-but nothing definite in the way of bands ? Are all these cases of burst cysts, or can there be tags of vitreous adherent to the retina, which are on the one hand sufficiently strong to cause traction and rupture of the retina, and on the other so fine as to be invisible with the ophthalmoscope ? It seems to be a matter which needs further investigation, for as we shall see in dealing next with the clinical significance of holes, it is a matter of the greatest importance to decide whether the retina has been "pushed in " or "pulled in."

We may hope that possibly in the future, examination of the vitreous with the slit lamp may give us valuable information on the point. 


\section{Clinical Significance of Holes in the Retina}

There is a very important fact, which Leber mentioned in his lectures at Heidelberg in 1882, but to which I think too little notice is given-namely that sudden detachment of the retina cannot occur without a hole.

In taking the history of cases with loss of sight in an eye, it is important but often difficult to distinguish between those in which defects in sight have existed for weeks or months but have been suddenly detected, from those where the loss of sight was actually sudden. The defect in an eye is so frequently only discovered on closing the other, and the individual is liable to think he has lost the sight suddenly, when in reality it has been defective for a long period, or possibly never has been good.

Sometimes, however, there is evidence in the history which distinguishes the two groups. A man may have noticed that one eye was uncomfortable or the sight impaired in some way, and have compared over some long period the sight in his two eyes, and then on a certain day - it may be, he can time the exact moment-the sight in one goes; such evidence is of great clinical significance especially in cases of detached retina.

With an intact eyeball and an intact retina, no detachment can take place unless there is a simultaneous and practically corresponding outpouring of fluid into the inter-retinal space on the one hand, and contraction of the vitreous on the other. Such pouring out of interretinal fluid and absorption of the vitreous cannot of course take place suddenly.

The onset of any detachment of the retina without a hole must therefore of necessity be gradual; whereas if there is a hole in the retina, transposition of fluid from the vitreous chamber through the hole into the inter-retinal space can readily take place, floating up the retina and causing a sudden loss of sight.

It would therefore appear that if it can be substantiated in any given case that the loss of sight from detachment was not merely suddenly detected, but actually occurred suddenly, we can definitely predict the presence of a hole, whether or not it can be seen by the ophthalmoscope.

A medical student was coming home late one night and was set upon by a lot of rough boys who knocked him down and hit him about the head and body. He did not notice that his eye specially had been hit, and being an intelligent and acute observer it is practically certain that he would have detected defect in the sight of either eye had any been present.

He, however, noticed nothing wrong with his eyes till eight days after, when, while walking up the outside steps of an omnibus, the sight suddenly failed in one eye. He was seen soon after at the 
London Hospital, and found to have a large detachment of the retina in which there was a big rent.

I think the reasonable explanation of his case is that one of the many blows he received fell on the eye and caused a hole in the retina, through which eight days later, on some sudden movement of the globe the vitreous passed and floated up the retina.

Transposition would certainly take place more easily when the vitreous is fluid-or when "detachment of the vitreous" has taken place and fluid fills the space between the contracted vitreous and the retina. Whether transposition of the normal jelly-like vitreous could take place through a hole is doubtful. Collins suggests that the reason why some holes are not associated with detachment is that in these the vitreous has notdegenerated and therefore is not fluid and does not pass through the hole.

A second point of clinical importance is that, so far as I am aware, no cases of detachment of the retina due to neoplasm, when examined pathologically, have been found associated with a hole in the retina. This is supported, so far as my own experience goes, by the fact that in every case of neoplasm the inter-retinal fluid sets solid on fixation, which I think must be a proof that there is no hole in the retina.

If it could be definitely proved that holes in the retina do not occur with a neoplasm, we should have a very important clue in the differential diagnosis of causes of detachment-namely, that if we find a hole in a detached retina we can exclude neoplasm.

It is unnecessary to labour the importance of such a diagnostic point for we are constantly seeing cases of detached retina in which there are no positive signs of neoplasm, but where we wonder whether "behind the veil" there lurks a malignant growth. Thus there may be no new vessels seen in the detached area, no abnormal pigmentation of the retina, no brown reflex on focal illumination, no shadow on transillumination and no congestion or pigmentation of the sclerotic seen externally. There may be no history of a blow, no myopia, no sign of cyclitis, either keratitis punctata or vitreous opacities, and no retinitis or choroiditis and yet perhaps the position of the detachment is one unaccounted for by gravity. Any safe clue to diagnosis would be welcomed. The presence of a-hole in the retina in such a case would, if we are correct in the above observation, give certain grounds for exclusion of a growth, and justification in leaving an otherwise suspicious eye unexcised.

To give an instance : a woman, aged about 50 , came to the hospital complaining of failure of sight, which had come on gradually. She had noticed a curtain, as it were, drawn across her sight from the outer side. On examination a large detachment was found on the nasal side and also below. In this case there was no history 
of blow, there was no myopia, no signs of cyclitis, retinitis or choroiditis, but there were some slight vitreous opacities. The detachment appeared quite "simple"; there were no new vessels or pigmentation of the retina, no sign of pigmentation or congestion of the sclerotic. The fact, however, that the detachment remained on the nasal side in a position unaccounted for by gravity, made me think there must be a growth on that side which was the cause of her trouble. I advised excision, but she refused to have the eye removed. It was not until a fortnight later that I found a large hole in the retina. I then felt justified in telling her that excision was unnecessary, and the subsequent history showed that this view, in her case at any rate, was correct. The eye gradually degenerated; it developed glaucoma, lost all perception of light and became painful. She then consented to have it removed. On pathological examination no growth was found.

I have recently seen another case of an elderly man who had a globular detachment limited to the upper part of the retina. There is no myopia, no history of a blow, no choroiditis or retinitis, no keratitis punctata, and only very slight vitreous opacity, but in the retina where it is detached there is a large rent, through which not only can one see the choroid but one has also the unusual opportunity of inspecting the posterior surface of the retina (Plate II). In this case, if there had not been a hole, one would have strongly suspected the presence of a growth.

To recapitulate the above remarks-I would suggest that (1) If we can detect a hole we can exclude growth, and (2) If we can substantiate a history of sudden loss of sight from detachment, even though a hole cannot be seen, we know that a hole must be present and we can with equal certainty exclude growth.

There is a third point of clinical importance which I should like to discuss, viz., the value of treatment of detachment of a retina in which there is a hole.

Treatment of detached retina (irrespective of the presence of a hole) is admittedly most trying to the individual and uncertain in its results, yet this must not of course deter one from enterprise in suitable cases, for when treatment is successful, the boon to the patient is inestimable. But I think we need to exercise the greatest care in determining which cases are suitable and which are not, in order that a good procedure may not be undervalued by the profession, and the public may not have its confidence in the profession undermined by unsuccessful treatment.

We need every help to group our cases into those in which operation holds out the possibility of success and those in which no treatment can succeed.

If we divide our cases of detachment as far as possible by their aetiology, we recognise that the retina is either- 
(1) Dragged in by an attached and shrinking vitreous, or by interstitial contraction as in retinitis striata.

(2) Pushed in by fluid poured out from the choroid into the interretinal space owing to some low form of inflammation.

or (3) It may be floated in by transposition of the vitreous through a retinal hole.

I. Where no hole in the detachment can be detected, I would suggest that-It is in the cases where we may reasonably suppose that the retina has been "pushed in" by fluid in the inter-retinal space-apart of course from cases of neoplasm - that we may hope for success in treatment.

Also in the cases in which we can find no cause for the separation of the retina, we may recommend treatment in the hope that a proportion may be benefited.

When however we can substantiate that the retina is being dragged in by vitreous bands or by cicatricial changes in its substance, no treatment at present devised is, as a rule, of any avail and should seldom be attempted : it is labour lost, a valueless strain is made on the patient and a needless postponement of adjustment to his limitations. Von Hippel's brilliant case, in which he divided a cicatricial band and the retina went back and useful vision was restored, is very exceptional, for a single definite band is of great rarity.

II. Where a hole can be detected in a detached retina, is treatment of any avail ? So far as my experience goes, when there is a hole in the retina, the detachment is usually extensive and the vision correspondingly poor; there are, however, exceptions. Thus (i) macular holes, as mentioned above, are for some reason seldom associated with much detachment. Why transposition of the vitreous through such holes does not usually occur is not so far as I know yet explained: possibly it is the jelly like condition of the vitreous or possibly some macular holes are not real holes but only cysts. (ii) Certain peripheral holes in the retina may also occasionally occur with no detachment, as in the case I have already mentioned, or with a detachment which remains strictly localised. I have recently seen a case where there was a small strictly localised detachment in the retina below, following trauma in which there was a large rent. This has remained stationary for four months and the eye has retained perfect acuity of central vision-6/6ths and Jaeger 1-with a practically complete field, there being only a slight contraction above. It is probable that here the retina is bound down by choroiditis at the base of the detachment, for there is a definite disturbance of the retinal and choroidal pigmentation in this region, and that it is for this reason the detachment remains stationary.

In such cases clearly no treatment is indicated. 
In the majority of cases of peripheral holes, however, detachment is extensive.

Leber considered that a hole in the retina was essential to its re-attachment, since through it the fluid in the inter-retinal space can pass back into the vitreous chamber and the retina become reseated. It is difficult to understand, however, if reseating of the retina takes place with rest alone, what is to maintain it there, and prevent its being floated up again directly the eye is moved, unless there is plastic choroiditis present to hold it in place, a very unlikely concomitant.

In the report of the Committee appointed by the Ophthalmological Society of the United Kingdom to collect data with regard to the cases of cure after detachment of the retina, three cases were mentioned in which there was a hole where cure was effected without operation; but in no case with hole was success obtained by means of operation.

Good results have been obtained by many surgeons, who after draining the inter-retinal space have punctured an intact retina; but this does not appear to me to be a true indication that cases of detachment with holes are responsive to treatment. There is surely a considerable difference between draining the inter-retinal space with subsequent puncturing of an intact retina and the attempt to drain the inter-retinal space where there already exists a retinal tear. In the former we may expect the inter-retinal space to be practically emptied of fluid, and the retina approaching the pigment epithelium before the puncture in the retina is made; the wound of the retina too might help in the glueing of the retina into position. Whereas if we attempt to drain an inter-retinal space with an already existing retinal tear, we should be draining the vitreous through the hole almost as much as draining the interretinal space.

I have heard of one case in which there was a hole, where the retina went back after operation, but the improvement was only temporary; the detachment subsequently reappeared.

Personally I have seen no case in which there was a hole improve either spontaneously or after treatment, and it is my opinion that with our present available methods such cases of detachment are practically hopeless. The experience of one man is however of little value, and in order to answer the question whether, with our present methods, treatment of cases of detachment with holes should be undertaken or not-we require-

(1). Increased knowledge of the causation of holes,

(2). Improved clinical methods of detecting whether the retina is being pushed in by fluid from without, or dragged in by adherent and contracting vitreous, and

(3). A larger number of reports of the practical experience of 
surgeons in the treatment of these cases, classified according to their cause.

Not until we have a fuller knowledge of their aetiology can we reasonably hope either to advise rationally or to devise a more successful procedure in the treatment of these difficult cases.

\title{
THE RELATION OF EXOPHORIA IN EARLY PRESBYOPIA TO REFRACTIVE ERRORS*
}

BY

\author{
Dr. Luther C. Peter \\ PHILAdelphia, Pa.
}

Routine studies in any particular line of cases are rewarded by the uncovering of many unsuspected, but none the less prevalent, conditions. The practice of routine muscle studies in refraction is no exception to the rule. One of the outstanding facts in this special line of investigation is the gradual increase in the number of exophorias encountered from the age of thirty to that of forty-five or fifty. Two distinct types of cases are to be found in this period. First are those which simply represent a carry-over, as it were, of an aggravated condition which has its inception in early adult life and increases with the added strain of waning accommodation in the presbyopic period. These fortunately make up the smaller group. From an aetiological standpoint, as well as from the standpoint of possibilities in treatment, they furnish a fascinating field for study. The second or larger group are those which one may appropriately call "accommodative exophorias" - exophorias which have as their basis failing accommodation. This group includes so many cases which need our careful consideration, that they alone will be discussed.

The underlying factor is failing accommodation. A summary, however, of case records seems to point to one fact especially significant, a majority of these patients have never worn glasses or have been improperly glassed until the age of thirty-five or later. If glasses are properly prescribed in early adult life, and again as the presbyopic age creeps on, this type of exophoria is comparatively infrequent in occurrence and is easily managed. In the writer's experience, those, who have most symptoms, have the greatest degrees of exophoria, and are the most difficult to handle, are the hypermetropes who have failed to wear suitable glasses until the early presbyopic period.

* Read before the Ophthalmic Section of the College of Physicians of Philadelphia, October, 1923. 